\title{
HRCT Imaging to Determine the Frequency of Anatomic Variations in Temporal Bone
}

\author{
Tanushree R Kamath ${ }^{1}$, Ram Shenoy Basti ${ }^{2}$ Mahesh $^{3}$ \\ ${ }^{1}$ Resident, Department of Radiodiagnosis, ${ }^{2}$ Professor, Department of Radiodiagnosis, ${ }^{3}$ Senior Resident, Department of \\ Radiodiagnosis, Father Muller Medical College, Kankanady, Mangalore, Karnataka, India
}

Corresponding author: Dr Tanushree R Kamath, 201-Poojan Pearl Apratments, Jodumatta Street, Mangalore - 575001, Karnataka, India

DOI: http://dx.doi.org/10.21276/ijcmsr.2019.4.4.20

How to cite this article: Tanushree R Kamath, Ram Shenoy Basti, Mahesh. HRCT imaging to determine the frequency of anatomic variations in temporal bone. International Journal of Contemporary Medicine Surgery and Radiology. 2019;4(4):D84-D86.

\section{A B S T R A C T}

Introduction: Surgical treatment is the main stay of treatment for various chronic ear pathologies. An ENT surgeon needs to have a precise knowledge of anatomy prior to surgery as each individual can have a varied anatomy and drastic complications like injury to nerve or vessel can be avoided or incomplete evacuation leading to recurrence of disease can be prevented Material and methods: Data was obtained retrospectively from 340 temporal bone high resolution computed tomography (HRCT) of 170 patients referred to Department of Radio diagnosis, Father Muller Medical College and Hospital for HRCT temporal bone scan. Anatomic variants such as facial nerve dehiscence (FND), dural exposure (DE), high jugular bulb (HJB), bulging / anteriorly placed sigmoid sinus, Koerner s septum (KS), aberrant internal carotid artery (AICA)was looked for and documented and frequency of each was estimated.

Results: 340 temporal bones of 170 patients were recruited in the study. HJB was encountered in 80 ears (23\%) and it was bilateral in 10 (5\%) patients. KS was seen in 90 ears (26\%)patients. Anteriorly placed sigmoid sinus in 125 ears (36\%), low hanging dura in 10 ears (2.9\%), aberrant internal carotid artery in 2 ears (0.5\%) of total 350 ears. FND was detected in 45 ears (13\%), 35 of them with cholesteatoma and 10 of them without cholesteatoma.

Conclusion: Radiologist must be well aware of important anatomical variants such as aberrant internal carotid artery, facial nerve dehiscence, low hanging dura, high jugular bulb, anterior placed sigmoid sinus and it's recognition and reporting prior to surgery is imperative. It decreases the possibility of surgical complications as well as surgical revision for recurrence. A preoperative warning about anatomical variant can help the surgeon to decide the best surgical approach.

Keywords: Facial Nerve Dehiscence (FND), Dural Exposure (DE), High Jugular Bulb (HJB), Koerner Septum (KS), Aberrant Internal Carotid Artery (AICA)

\section{INTRODUCTION}

Surgical treatment is the main stay of treatment for various chronic ear pathologies. An ENT surgeon needs to have a precise knowledge of anatomy prior to surgery as each individual can have a varied anatomy and drastic complications like injury to nerve or vessel can be avoided or incomplete evacuation leading to recurrence of disease can be prevented.

Facial nerve dehiscence is not uncommon and mostly occurs in the tympanic segment near the oval window. The horizontal segment at times is displaced inferiorly to cover the oval window or lies exposed over the promontory. ${ }^{1}$

The tegmen of the mastoid and attic passes usually in a horizontal plane slightly lower than the arcuate eminence produced by the top of the superior semicircular canal. A depression of the tegmental plate can cause low hanging dura which may cover the roof of the external auditory canal which then will have risk of penetration of the cranial cavity during surgery.
There are various methods to define a high jugular bulb, for example jugular bulb is usually below the level of floor of IAC or EAC or basal turn of cochlea or floor of hypo-tympanum. An uncovered jugular bulb/ dehiscent bulb is much more serious as it can be mistaken for a glomus tumor on otoscopy or it may be injured during surgery.

The sigmoid sinus forms a shallow indentation on the posterior aspect of mastoid. It is an important landmark in mastoidectomy surgery and drilling is done at this level. In case of anteriorly placed sinus or bulging sinus it courses more anteriorly and produces a bulge in the mastoid, best seen in the axial sections. This is important as the distance between sigmoid sinus to the posterior wall of the external auditory canal determines the amount of space available for the postaural approach to the mastoid antrum.

Körner's septum (KS) refers to a dense, bony plate found in the mastoid which represents the persistence of the petrosquamous suture line. This septum divides the mastoid process into a superficial squamous portion and a deep petrous 
portion. If it is present and not picked up preoperatively it leads to incomplete evacuation of disease and recurrence An aberrant internal carotid artery (AICA) is a rare vascular anomaly taking an aberrant lateral course in the temporal bone and passes through the middle ear cavity. ${ }^{1}$ It will pass along the medial aspect of the middle ear coursing anteriorly across cochlear promontory to join the horizontal carotid canal through a dehiscence in the carotid plate. ${ }^{2}$ It is due to collateral pathway that occurs as a result of agenesis of first embryonic segment of the ICA.

\section{MATERIAL AND METHODS}

This study was an observational descriptive chart based study conducted retrospectively with data retrieval period of 6 months.

Inclusion criteria: Patients who underwent HRCT temporal bone

Exclusion criteria: Previously operated cases where anatomy is distorted

Ethical clearance for the study was obtained by the institution research and ethical committee. Data was obtained retrospectively from 340 temporal bone high resolution computed tomography (HRCT) of 170 patients referred to Department of Radio diagnosis, Father Muller Medical College and Hospital for HRCT temporal bone scan.

HRCT temporal bone was performed using GE BRIGHT SPEED 16 SLICE as per the department protocol. Scans were obtained in the helical mode to lower motion artefacts. Scanning parameters of $120 \mathrm{kV}, 150 \mathrm{mAs}, 1 \mathrm{~mm}$ section thickness were taken. HRCT was done using thin section high resolution and bone algorithm technique reconstruction. Coronal and sagittal reformatting was done to a slice thickness of $0.625 \mathrm{~mm}$.

In case of bilateral temporal bone pathologies, each side was taken as a separate case. HRCT images were evaluated in detail and findings tabulated. Anatomic variants such as facial nerve dehiscence (FND), dural exposure (DE), high jugular bulb (HJB), bulging / anteriorly placed sigmoid sinus, Koerners septum (KS), aberrant internal carotid artery were looked for and frequency of anatomic variant was determined.

\section{RESULTS}

340 temporal bones of 170 patients were recruited in the study. HJB was encountered in 80 ears $(23 \%)$ and it was bilateral

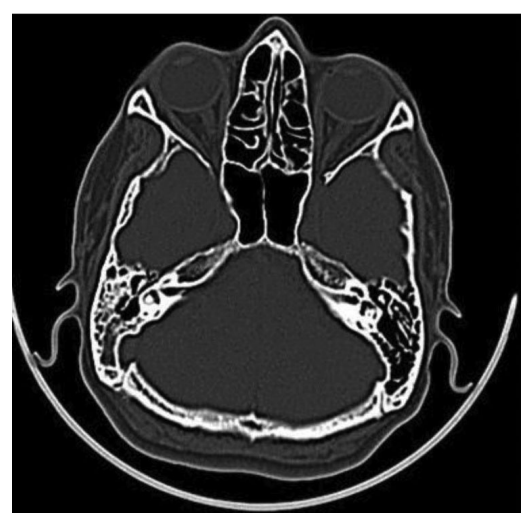

Figure-1: Right Korners t

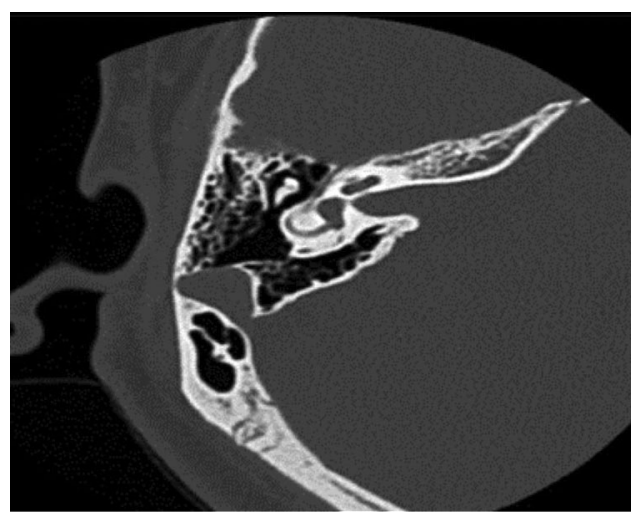

Figure-2: Bulging or anteriorly placed

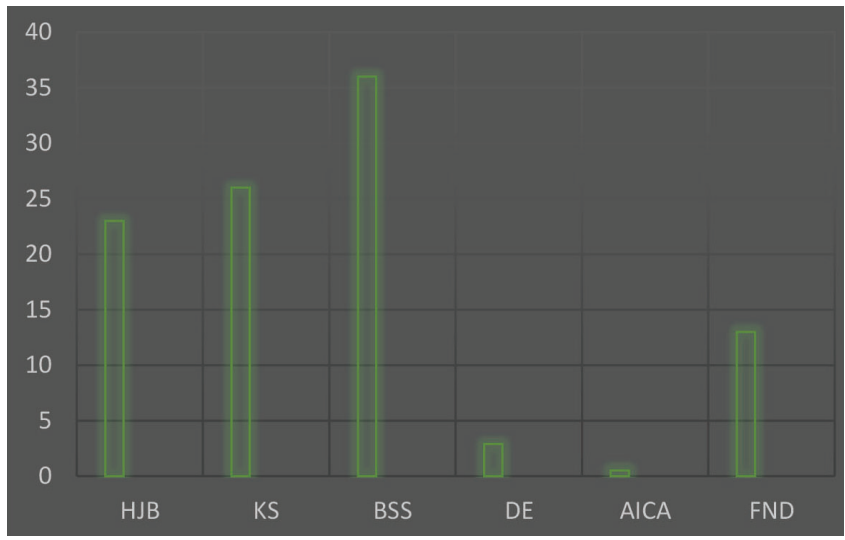

Graph-1: Percentage frequency of anatomic variant

in $10(5 \%)$ patients. KS was seen in 90 ears (26\%) patients (graph-1). Anteriorly placed sigmoid sinus in 125 ears (36\%), low hanging dura in 10 ears $(2.9 \%)$, aberrant internal carotid artery in 2 ears $(0.5 \%)$ of total 350 ears. FND was detected in 45 ears (13\%), 35 of them with cholesteatoma and 10 of them without cholesteatoma.

\section{DISCUSSION}

High-resolution computed tomography (HRCT) is the main modality for the investigation of the ear diseases, to visualize extent of disease and for preoperative planning. It provides a good amount of info regarding the extent, the bony erosion which is diagnostic in case of cholesteatoma, and any significant anatomical variant which can cause disastrous consequence intraoperatively or lead to recurrence. HRCT should be made a routine preoperative investigation in case of mastoid or ear surgeries.

Koesling et al. found a low frequency of $6 \%$ for $\mathrm{HJB}^{3}$ and Karaca et al found a frequency of $32 \%{ }^{4}$

According to Wiet et al operative facial paralysis is the second most common reason for malpractice in ear surgery today in many common otologic approaches. ${ }^{5}$ The frequency of FND varies from $0.5 \%$ to $74 \%$ in the literature. ${ }^{6}$

Park et al. found a strong correlation between radiological assessment and surgical findings in the sigmoid sinus position. ${ }^{7}$

According to Alam et al both radiological and surgical assessment confirmed the presence of Korner's septum in $88 \%$ of patients. ${ }^{8}$ Radiological assessment revealed normal position of dura in $76 \%$ patients and low lying dura in $24 \%$ 
patients whereas surgical evaluation revealed normal dura in $78 \%$ and low dura in $22 \%$ only. ${ }^{8}$

AICA in the middle ear is a rare congenital abnormality usually identified on CT imaging. According to Karaca et al frequency of AICA was only $0.02 \%{ }^{4}$

According to Zhang et al there is a good radio-surgical correlation in chronic otitis media for most middle ear structures so HRCT scan should be a routine examination prior to middle ear and mastoid surgery. ${ }^{9}$

\section{CONCLUSION}

Radiologist must be well aware of important anatomical variants such as aberrant internal carotid artery, facial nerve dehiscence, low hanging dura, high jugular bulb, anterior placed sigmoid sinus and it's recognition and reporting prior to surgery is imperative. It decreases the possibility of surgical complications as well as surgical revision for recurrence. A preoperative warning about anatomical variant can help the surgeon to decide the best surgical approach.

\section{REFERENCES}

1. Zaghouani H, Benzina N, Zarrad CH, Kermani W, Limeme M, Majdoub S, Amara H, Bakir D, Kraeim C. Imaging of anatomical variations of the temporal bone to specify to the surgeon before middle ear surgery. European Congress of Radiology 2013.

2. Reis CVC, Deshmukh V, Zabramski JM, Crusius M, Deshmukh P, Preul MC, et al. Anatomy of the mastoid emissary vein and venous system of the posterior neck region: Neurosurgical implications. Neurosurgery 2007;61(2):193-201.

3. Koesling S, Kunkel P, Schul T. Vascular anomalies, sutures and small canals of the temporal bone on axial CT. European journal of radiology. 2005;54(3):335-43.

4. Karaca CT, Toros SZ, Noseri HK. Analysis of anatomic variations in temporal bone by radiology. The Journal of International Advanced Otology. 2012;8(2):239.

5. Wiet RJ. Iatrogenic facial paralysis. Otolaryngologic clinics of North America. 1982;15(4):773-80.

6. Wang HM, Lin JC, Lee KW, Tai CF, Wang LF, Chang HM, Hsu YC, Chai CY, Ho KY. Analysis of mastoid findings at surgery to treat middle ear cholesteatoma. Archives of Otolaryngology-Head \& Neck Surgery. 2006;132(12):1307-10.

7. Park E, Amoodi H, Kuthubutheen J, Chen JM, Nedzelski JM, Lin VY. Predictors of round window accessibility for adult cochlear implantation based on pre-operative CT scan: A prospective observational study. J Otolaryngol Head Neck Surg 2015;44(5):20.

8. Alam-Eldeen MH, Rashad UM. Radiological requirements for surgical planning in cochlear implant candidates. The Indian journal of radiology \& imaging. 2017;27(3):274.

9. Zhang X, Chen Y, Liu Q,Han Z, Li X. The role of highresolution $\mathrm{CT}$ in the preoperative assessment of chronic otitis media. Lin chuang er bi yan hou ke za zhi= Journal of clinical otorhinolaryngology. 2004;18(7):396-8.

Source of Support: Nil; Conflict of Interest: None

Submitted: 26-08-2019; Accepted: 09-10-2019; Published online: 24-11-2019 